\title{
OBJECT SEGMENTATION USING MULTISCALE MORPHOLOGICAL OPERATIONS
}

\author{
Dr. A. Srikrishna ${ }^{1}$, P. Pallavi ${ }^{2}$, V. Geetha Madhuri ${ }^{3}$, N. Neelima ${ }^{4}$ \\ 1,2,3,4 Department of Information Technology, \\ RVR \& JC College of Engineering, \\ Chowdavaram, Guntur, Andhra Pradesh, India \\ atlurisrikrishna@yahoo.com \\ paladugupallavi3egmail.com \\ madhurigeetha33@gmail.com \\ neelima_nalla@yahoo.co.in
}

\begin{abstract}
Object segmentation plays an important role in human visual perception, medical image processing and content based image retrieval. It provides information for recognition and interpretation. This paper uses mathematical morphology for image segmentation. Object segmentation is difficult because one usually does not know a priori what type of object exists in an image, how many different shapes are there and what regions the image has. To carryout discrimination and segmentation several innovative segmentation methods, based on morphology are proposed. The present study proposes segmentation method based on multiscale morphological reconstructions. Various sizes of structuring elements have been used to segment simple and complex shapes. It enhances local boundaries that may lead to improve segmentation accuracy. The method is tested on various datasets and results shows that it can be used for both interactive and automatic segmentation.
\end{abstract}

\section{KEYWORDS}

Morphology, Structuring Element, Segmentation, Edge Detection, Skeletanization

\section{INTRODUCTION}

Humans recognize various objects in an image though the objects may vary somewhat in different viewpoints and on various transformations. Object segmentation is useful task in object recognition. The object recognition determines an object in a given set of objects in an image or image sequence. In order to perform object recognition the objects from a give image or image sequence are to be identified. For this object segmentation that is to distinguish objects from background is performed.

Object segmentation [3] is the process of partitioning a digital image into multiple segments (sets of pixels, also known as super pixels). The goal of segmentation is to simplify and/or change the representation of an image into something that is more meaningful and easier to analyze. Object segmentation is typically used to locate objects and boundaries (lines, curves, etc.) in images. More precisely, object segmentation is the process of assigning a label to every pixel in an image such that pixels with the same label share certain visual characteristics.

Some of the applications of object segmentation are content based image retrieval, machine vision, medical imaging, object detection, recognition tasks, traffic control systems, video

DOI : 10.5121/ijcseit.2013.3402 
surveillance. Object segmentation is the partition of an image into a set of non overlapping regions whose union is the entire image. The purpose of segmentation is to decompose the image into part that is meaningful with respect to a particular application. Concerning visual signal processing, image segmentation is essential for various applications. It describes the process whereby each pixel in an image is labeled, such that pixels with the same label present coherent visual characteristics. This allow for a semantic approach to image analysis. One way to perform image segmentation is to simply utilize the clustering algorithm in the color space domain [1], i.e., HSV or RGB; segmentation can also be based on the statistics of the color space description of the image, e.g., color histogram. These methods are carried out in the color space domain instead of the image pixel domain, whose results depend on the initial cluster setting. Edge-based segmentation is simple but it requires a further linking procedure to segment an image [2], [3], [4]. Among color region-based approaches, the region-growing approach [5] provides an initial set of seeds; regions are then grown by comparing neighboring pixels, which are merged [6] with the region with the closest mean color. JSEG [7], [8], [9], [10] seeks to divide an image into spatially continuous disjoint and homogenous regions based on the image. It uses a region merging approach, but the color information between entire neighboring regions, rather than individual pixels, is utilized. Experiments show that JSEG provides satisfactory results on most color images. The watershed technique splits one image into regions based on its gray-level topology and is performed on the gradient image. Regions are split by watersheds, which are constructed from adjacent catchment basins. Although it has the advantage of being able to segment regions with closed contours, it suffers from over segmentation and requires region merging processing afterward. Multiscale morphological reconstruction [11] is used to eliminate the over segmentation in the watershed algorithm. Graph based segmentation [12], [13] takes the global image information and local spatial relationships into consideration to perform image segmentation. It defines a predicate to measure the boundary evidence between two neighboring regions to yield a graph-based representation of one image.

The general-purpose segmentation algorithms [1], [7], [8], [9], [10], [12], [13] represent one image with disjoint regions of homogeneous color/texture features for higher level applications, and the object segmentation ones [11], [14] extract image objects with different gray level variations and noise attacks. The former does not address object segmentation from its design target. The latter focuses on segmenting the object of different scale but do not perform parameter adaptation when dealing with images with different background (BG) variation and object contents. To utilize both region and object-based segmentation capabilities to handle the object segmentation for large-scale database images in a robust and principled manner an algorithm is proposed. The proposed algorithm is known as object segmentation using multiscale morphology (OSMM). Morphological open (close) by reconstruction [14], [15], i.e., OR (CR), with a proper structure element (SE) on the gray levels, to automatically segment images' object region is used. Section 2 discusses the basic terminology of mathematical morphology. Section 3 discusses segmentation using multiscale morphology. Section 4 deals with results and discussions.

\section{BASIC TERminology OF MATHEMATICAL MORPHOLOGY}

\subsection{Mathematical Morphological Operations}

\subsubsection{Dilation}

Dilation is one of the elementary operators of mathematical morphology, that is, it is a building block for a large class of operators. The key process in the dilation operator is the local comparison of a shape, called structural element, with the object to be transformed. When the structural element is positioned at a given point and it touches the object, then this point will appear in the result of the transformation, otherwise it will not. In dilation the value of the output 
pixel is the maximum value of all the pixels in the input pixel's neighbourhood. In a binary image, if any of the pixels is set to the value 1, the output pixel is set to 1 . The dilation of a gray level image $I(x, y)$ by two-dimensional structuring element $B$ is defined as follows

$$
(I \oplus B)(x, y)=\max \{I(x-k, y-l) \mid(k, l) \in B\}
$$

\subsubsection{Erosion}

Erosion is one of two fundamental operations (the other being dilation) in morphological image processing from which all other morphological operations are defined. It was originally defined for binary images, later being extended to gray scale images, and subsequently to complete lattices.

The erosion is one of the elementary operators of mathematical morphology, that is, it is one of the building blocks of a large class of operators. The key mechanism under the erosion operator is the local comparison of a shape, called structural element, with the object that will be transformed. If, when positioned at a given point, the structural element is included in the object then this point will appear in the result of the transformation, otherwise not. The value of the output pixel is the minimum value of all the pixels in the input pixel's neighbourhood. The erosion of a gray level image $I(x, y)$ by two dimensional structuring element $B$ is defined as follows

$$
(I \ominus R)(x, y)=\min \{I(x+k, y+l) \mid(k, l) \Leftarrow R\}
$$

\subsubsection{Opening}

Opening smoothes the contour of an object, breaks narrow isthmuses, and eliminates thin protrusions. The opening of $I$ by structuring element $B$ is obtained by the erosion of $I$ by $B$, followed by dilation of the resulting image by $B$ is denoted by $I \circ B$ which is given in the form of equation as follows

$$
I \circ B=(I \ominus B) \oplus B
$$

\subsubsection{Closing}

Closing tends to smooth sections of contours but, as opposed to opening, it generally fuses narrow breaks and long thin gulfs, eliminates small holes, and fills gaps in the contour. The closing of $I$ by $B$ is obtained by the dilation of $I$ by $B$, followed by erosion of the resulting structure by $B$ is denoted by $I \bullet B$ which is given in the form of equation as follows

$$
I \bullet B=(I \oplus B) \ominus B
$$

\subsection{Multiscale Morphological Operations}

Let the structuring element $B$ defined in Equations 1, 2, 3, 4 be $n . b$ where $b$ denotes the smallest structuring element size in the discrete domain. The $n^{\text {th }}$ homothetic of a convex structuring element $b$ can be obtained by dilating $b$ recursively $n-1$ times with itself given by Equation 5 .

$$
n \cdot b=b \oplus b \oplus b \oplus \ldots . . \oplus b \quad(n-1) \text { times }
$$


By controlling $n$, the multiscale morphological operations decompose one image into a set of filtered images. These operations are self-calibrated in which the filtered image produced by a structuring element of a particular scale should strictly contain only the features of that scale.

Multiscale morphological reconstruction operations on gray-level images can be applied in a dual approach to segment objects. Let $g=I \circ B$, and the morphological open by reconstruction (OR) can be defined by Equation 6 .

Where

$$
I \widetilde{\sigma}_{B}=I_{B}^{O R(n)}=\delta_{b}^{n+1}(g, I)
$$

$$
\begin{aligned}
& \delta_{b}^{1}(g, I)=\min (g \Phi b, I), \delta_{b}^{j+1}(h, I)=\min \left(\delta_{b}^{j}(g, I) \Phi b, I\right) \text { and } n \text { is an integer } \\
& \text { such that } \delta_{b}^{n+1}(g, I)=\delta_{b}^{n}(g, I) .
\end{aligned}
$$

Similarly, let $h=I \bullet B$ the morphological close by reconstruction (CR) can be defined by Equation 7.

$$
I \widetilde{\bullet}_{B}=I_{B}^{C R(m)}=\epsilon_{b}^{m+1}(h, I)
$$

where

$\varepsilon_{b}^{1}(h, I)=\max (h \ominus b, I), \varepsilon_{z}^{j+1}(h, I)=\max \left(\epsilon_{b}^{j}(h, I) \ominus b, I\right)$ and $m$ is an integer such that $\varepsilon_{b}^{m+1}(h, I)=\epsilon_{b}^{m}(h, I)$.

\section{Segmentation using Multiscale Morphology}

The general-purpose segmentation algorithms such as mean shift segmentation, edge based segmentation represent one image with disjoint regions of homogeneous color or texture features for higher level applications, and the object segmentation ones such as watershed segmentation, graph based segmentation extract image objects with different gray level variations and noise attacks. The former does not address object segmentation from its design target. The latter focuses on segmenting the object of different scale but do not perform parameter adaptation when dealing with images with different background (BG) variation and object contents. In order to overcome the drawbacks, segmentation using multiscale morphology has been proposed. This method utilizes both region- and object-based segmentation capabilities to handle the object segmentation in a robust and principled manner. Figure 1 shows the block diagram of the object segmentation using multiscale morphological reconstructions.

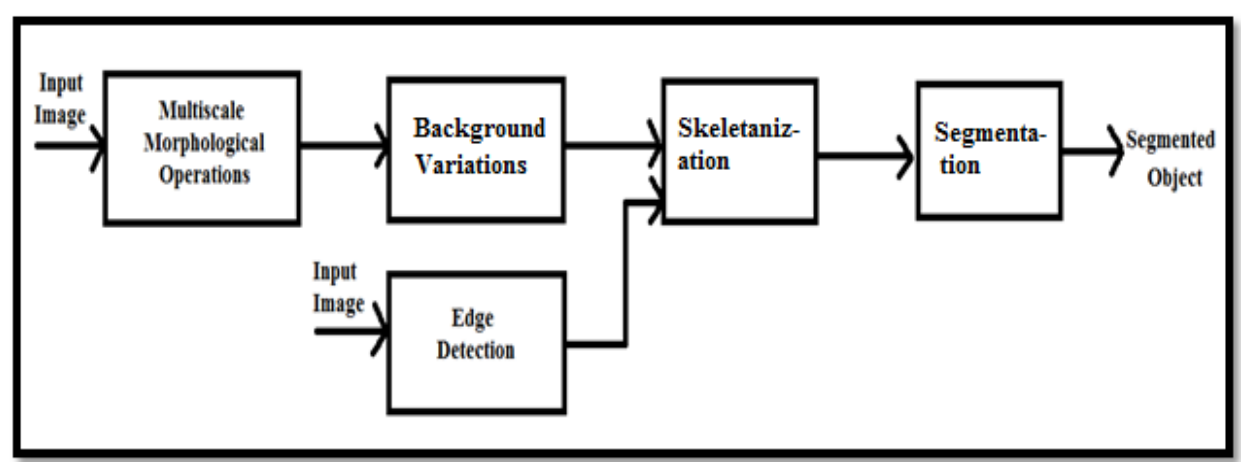

Figure 1. Object Segmentation using Multiscale Morphology. 


\subsection{Background Variations}

Open by reconstruction (OR),close by reconstruction (CR) and top- (bottom-) hat operations are used to segment object regions $I_{o}^{\alpha}$ and their gray levels $I_{o}=I \wedge I_{O}^{\alpha}$, where $\Lambda$ is a binary AND operation. The reconstruction operations of OR (CR) would not be fully iterated for stable outcome so that convex (concave) gray-level variation regions can be located. The image processed by this partial OR (CR) operation is denoted as $I_{B}^{O R(n-)}$ with $n^{-}<n$ to be distinguished from the fully reconstructed one in that requires $n$ iterations. The basic idea is images with identifiable back ground regions (BGs) usually present homogeneously evolving gray-level BGs, i.e. $(\partial f / \partial x)$, $(\partial f / \partial y)$ is continuous. When performing gray-level open by reconstruction (OR) operations on image with suitable structuring element sizes most background (BG) regions would coincide with the processed image $I_{E}^{O R(n)}$. To precisely locate back ground regions (BGs) the structuring element size in $I_{B}^{O R(n-)}$ should be properly selected such that object boundaries are identifiable. For precisely locating the structuring element, structuring element size is gradually enlarged and the frame difference of gray levels between $I$ and $I_{B}^{O R(n-)}$ i.e. $\Delta I_{B}^{O R(n-)}$ which is given by Equation 8 is calculated ( difference of gray levels for consecutive structuring elements is calculated).

$$
\Delta I_{B}^{O R(n-)}=\left|I-I_{B}^{O R(n-)}\right|=\sum_{x, y}\left|J(x, y)-I_{B}^{O R(n-)}(x, y)\right|
$$

The threshold value is calculated by using the Equation 9

$$
\text { threshold }{ }_{B}^{n-}=\Delta I_{B}^{O R(n-)}-\Delta I_{B}^{O R\left(n^{-}-1\right)} / a \times b
$$

where $a \times b$ is the size of the image.

To find a proper structuring element $B$ such that it yields a nearly stable gray-level variation, i.e., threshold given by Equation 9 should nearly approach zero. The proper $B$ has threshold given by Equation 9 values between 0 and 1 , and different images would require different $B_{C}\left(B_{C}\right)$ to achieve the aforementioned stability due to differing object contents.

\subsection{Edge Detection}

The edge pixels of the input image $I$ are detected by using morphological operations. The edge pixels denoted by $\operatorname{Edge}(I)$ for an image $I$ is given by the Equation 10

$$
\operatorname{Edge}(I)=\operatorname{Dilation}(I)-\operatorname{Erosion}(I)
$$

where Dilation $(I)$, Erosion $(I)$ are dilation, erosion operations on input image $I$ as defined in Equations 1, 2 respectively.

\subsection{Skeletanization}

The skeleton of an object $L(\mathrm{I})$ can be obtained by edge image and background variations. The skeleton obtained using skeletanization process is given by Equation 11 .

$$
L(I)=E d g e(I) \& \& \Delta I_{B}^{O R(n-)}
$$




\subsection{Segmentation}

The skeleton obtained using skeletanization process is given as the input for reconstruction of object. In the reconstruction the input image is dilated by varying sizes of structuring element $B$ which satisfies the condition using threshold value given by Equation 9 which is in the range of 0 and 1. Let $C_{1}, C_{2}, C_{3}, \ldots ., C_{n-}$ be the outputs of dilation for varying sizes of structuring elements. Then the segmented object is given by Equation 12 .

$$
\text { Segmented object }=C_{1}\left\|C_{2}\right\| C_{3}\|\cdots\| C_{n^{-}}
$$

The overall process of segmentation is given in the Algorithm

\subsection{Algorithm Object Segmentation using Multiscale Morphology (OSMM)}

Step 1: Read the input image.

Step 2: Convert the image into gray scale image.

Step 3: Perform "opening(erosion followed by dilation) or closing (dilation followed by erosion)" of the input image using varying sizes of structuring elements.

Step 4: The structuring element size is gradually enlarged and the frame difference of gray levels between $I$ and $I_{B}^{O R(n-)}$ i.e. $\Delta I_{B}^{O R(n-)}$ which is given by Equation 8 is recorded.

Step 5: Calculate the threshold by using the formula in Equation 9.

Step 6: The structuring elements whose threshold values are between 0 and 1 are stored.

Step 7: Calculate the edge pixels of the input image by using the formula in Equation 10.

Step 8: Perform detaching process given by Equation 11 .

Step 9: Perform reconstruction of segmented by using Equation 12.

\section{RESUlTS AND DiSCUSSIONS}

We evaluate shape prototypes in the context of object segmentation. These object segmentation techniques are used as a pre processing step for object recognition. We begin with a set of 200 objects, representing distinct views of collection of 101 objects from Caltech101 database. We are using this step as a pre processing step for object recognition so the input image consists of only one object.

To evaluate the performance of the algorithm correctness and completeness criteria are considered which are defined by Equations 13 and 14 respectively.

Correctness can be defined as the percentage of correctly extracted region (ground truth) by the segmentation algorithm and can be calculated using Equation 13.

$$
\text { correctness }=\frac{T P}{T P+F P} * 100 \%
$$

Completeness can be defined as the percentage of the ground truth region extracted by the segmentation algorithm and can be calculated using Equation 14.

$$
\text { completeness }=\frac{T P}{T P+F N} * 100 \%
$$


where to obtain the true positive (TP) image a logical AND operation was performed between the ground truth and the resultant image. The difference between the ground truth image and the true positive image was taken as the false negative (FN) image of the respective segmented image. The difference between the segmented image and the true positive image was taken as the false positive (FP) image of the respective segmented image. The TP, FP, FN are illustrated in Figure 2. Here we consider the ground truth which is a manually segmented image. The ground truth images are compared with the image segmented with the proposed algorithm. The Figure 3 to Figure 8 shows that the segmented images using proposed algorithm are very similar to ground truth. The objective analysis of the segment is evaluated using correctness and completeness which is shown from Table 1 to Table 6 . The correctness is above $80 \%$ in most of the cases and completeness is above $90 \%$ in all cases.

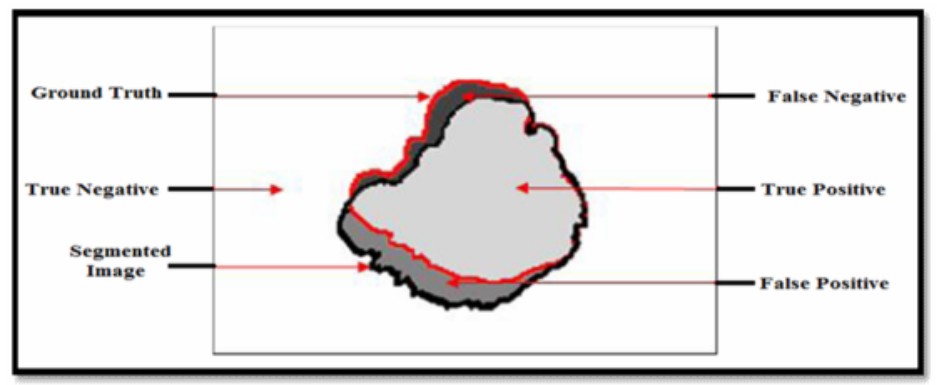

Lilly.png

Figure 2. Ground truth region of object and segmented region

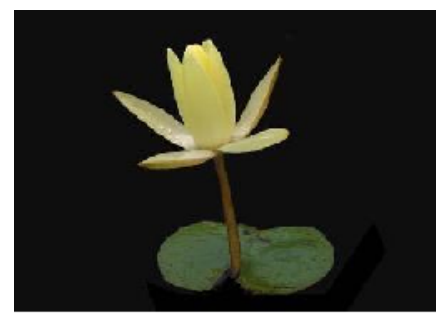

a. Original Image

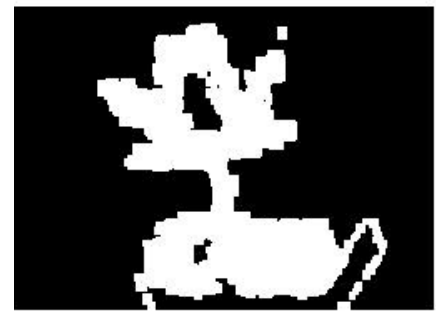

A Ontput for $7 \times 7$ sthe:huring clement

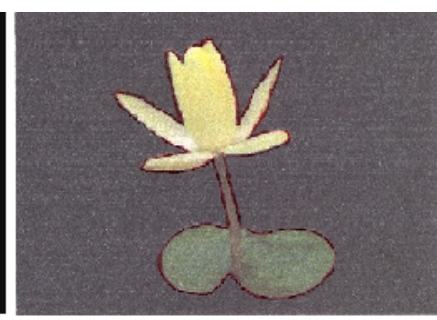

b. Ground Truth

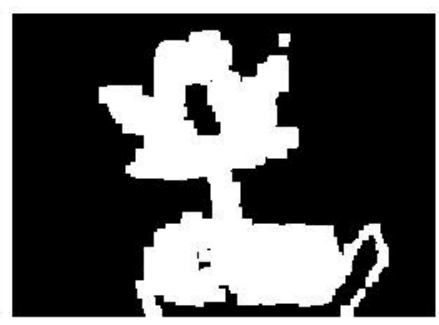

e. Output for $9 \times 9$ structuring element

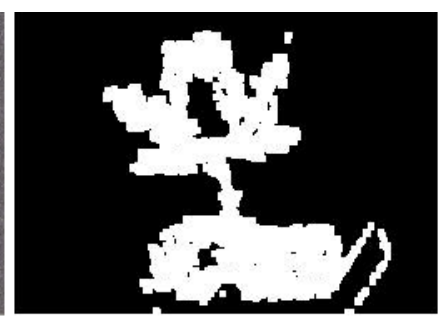

c. Oulpul fur $5 \times 5$ structuring

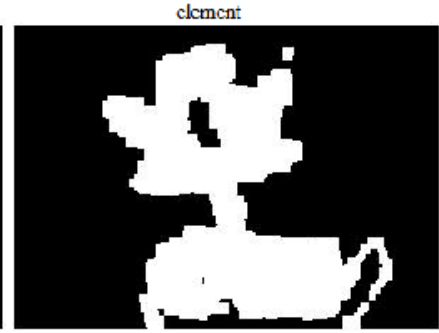

f. Output for $11 \times 11$ structuring element

Figure 3. a. Shows the Lilly image. b. Shows the ground truth which is taken manually. c-f represent the output of the image with varying structuring elements. 
International Journal of Computer Science, Engineering and Information Technology (IJCSEIT), Vol.3,No.4,August 2013

Table 1. Correctness and Completeness values for various sizes of structuring elements for Lilly.png.

\begin{tabular}{|c|c|c|}
\hline $\begin{array}{c}\text { Structuring Element } \\
\text { Size }\end{array}$ & Correctness & Completeness \\
\hline $5 \times 5$ & 86.8629 & 90.5704 \\
\hline $7 \times 7$ & 81.7156 & 93.7795 \\
\hline $9 \times 9$ & 77.2008 & 95.2278 \\
\hline $11 \times 11$ & 75.0547 & 96.3634 \\
\hline $13 \times 13$ & 72.6632 & 97.4097 \\
\hline $17 \times 17$ & 67.6016 & 98.7240 \\
\hline
\end{tabular}

Scissors.jpg

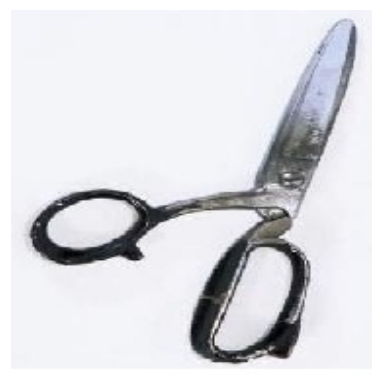

a. Urigirial lrnage

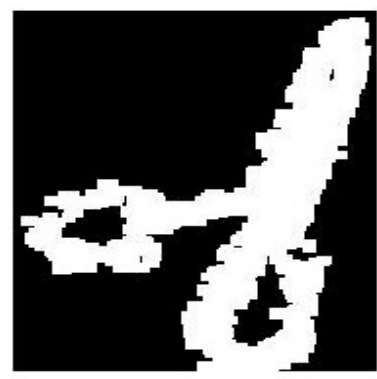

d. Output for $9 \times 9$ structuring elemenil

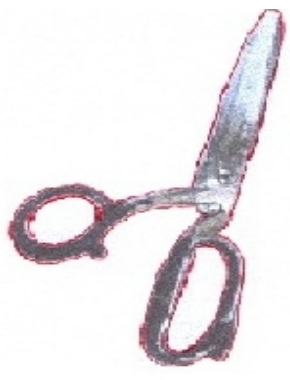

b Ciround Truth

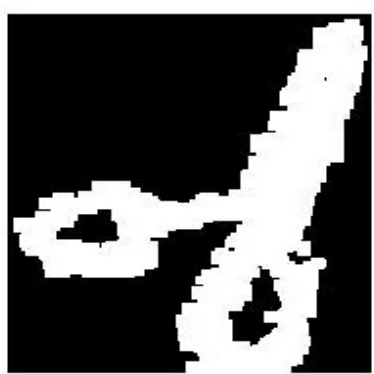

e. Output for $11 \times 11$

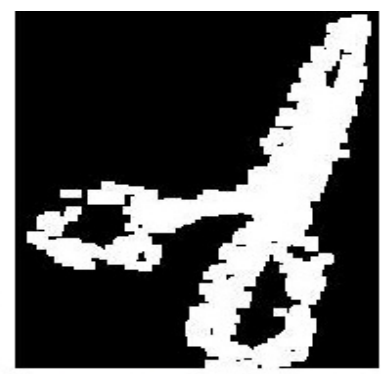

c. Output for $7 \times 7$ structuring

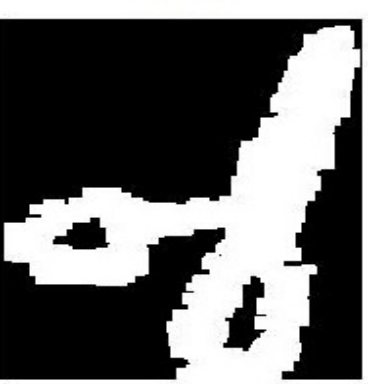
f. Uutput for $13 \times 13$ structuring

Figure 4. a. Shows the Scissors image. b. Shows the ground truth which is taken manually. c-f represent the output of the image with varying structuring elements

Table 2. Correctness and Completeness values for various sizes of structuring elements for Scissors.jpg

\begin{tabular}{|c|c|c|}
\hline $\begin{array}{c}\text { Structuring } \\
\text { Element } \\
\text { Size }\end{array}$ & $\begin{array}{c}\text { Correctnes } \\
\mathbf{s}\end{array}$ & $\begin{array}{c}\text { Completenes } \\
\mathbf{s}\end{array}$ \\
\hline $7 \times 7$ & 86.3627 & 79.4165 \\
\hline $9 \times 9$ & 82.1526 & 85.8422 \\
\hline $11 \times 11$ & 79.8946 & 88.6663 \\
\hline $13 \times 13$ & 78.5122 & 91.3224 \\
\hline $15 \times 15$ & 78.129 & 95.0174 \\
\hline $17 \times 17$ & 74.5465 & 97.4122 \\
\hline
\end{tabular}




\section{Mandolin.jpg}

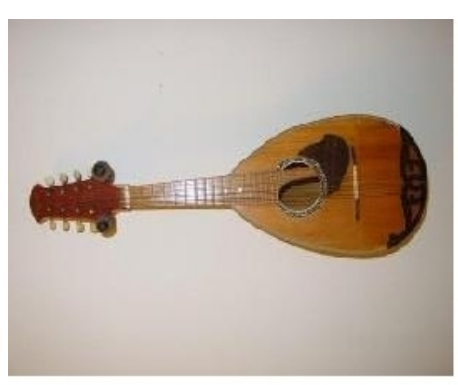

a. Original Image

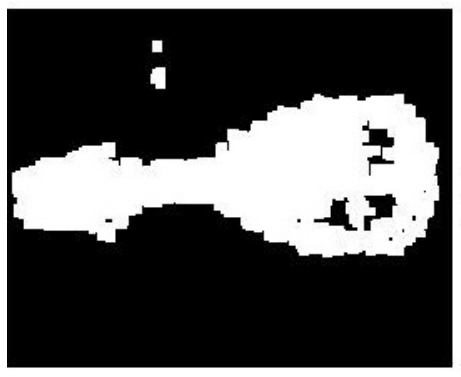

d. Output for $7 \times 7$ structuring

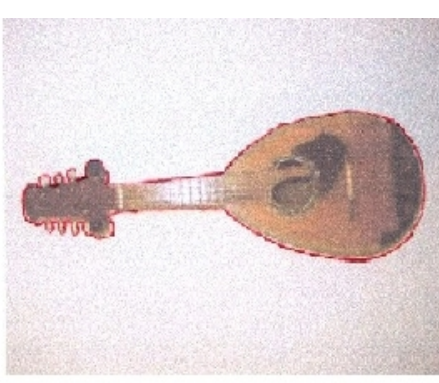

b. Ground Truth

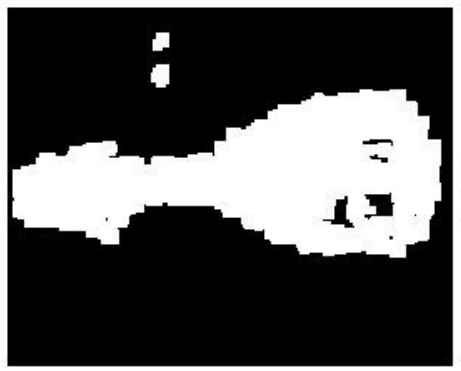

e. Output for $9 \times 9$ structuring clement

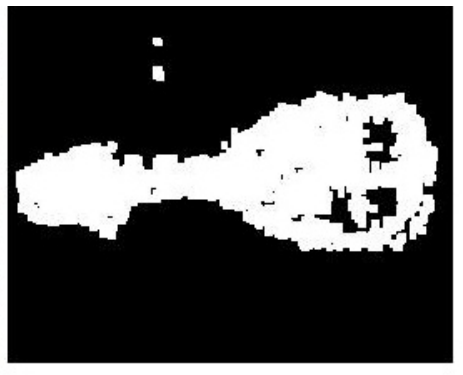

c. Output for $5 \times 5$ structuring element

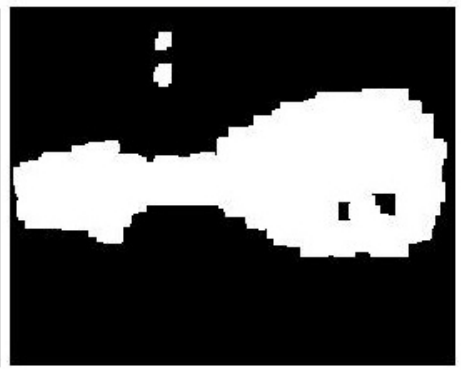

f. Output for $11 \times 11$ structuring element

Figure 5. a. Shows the Mandolin image. b. Shows the ground truth which is taken manually. c-f represent the output of the image with varying structuring elements.

Table 3. Correctness and Completeness values for various sizes of structuring elements for Mandolin.jpg

\begin{tabular}{|c|c|c|}
\hline $\begin{array}{c}\text { Structuring } \\
\text { Element } \\
\text { Size }\end{array}$ & $\begin{array}{c}\text { Correctnes } \\
\mathbf{s}\end{array}$ & $\begin{array}{c}\text { Completenes } \\
\mathbf{s}\end{array}$ \\
\hline $5 \times 5$ & 91.1111 & 90.4906 \\
\hline $7 \times 7$ & 86.8741 & 94.5327 \\
\hline $9 \times 9$ & 83.3252 & 96.9289 \\
\hline $11 \times 11$ & 81.4175 & 98.4424 \\
\hline $13 \times 13$ & 78.5678 & 99.1486 \\
\hline $15 \times 15$ & 75.0938 & 99.6657 \\
\hline
\end{tabular}


Seahorse.jpg

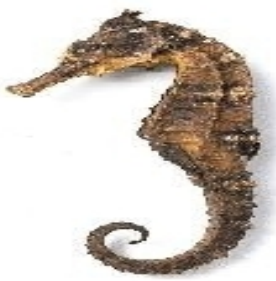

a. Criginal Imase

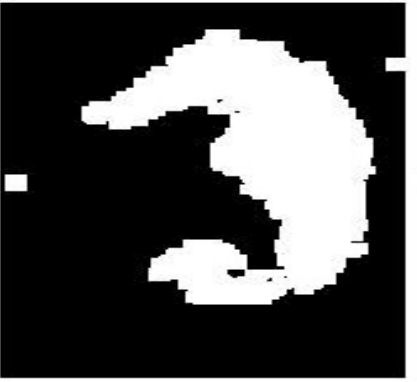

d. Output for 9xy structuring element

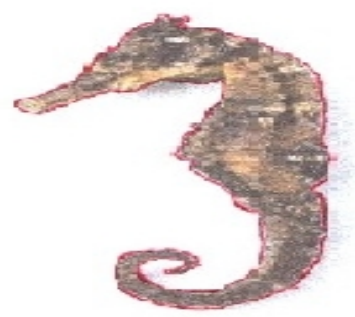

b. Ground Truth

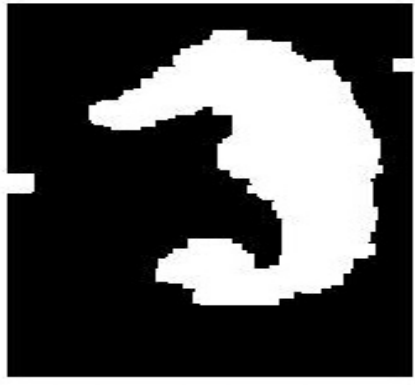

e. Output for $11 \times 11$ strucluring element

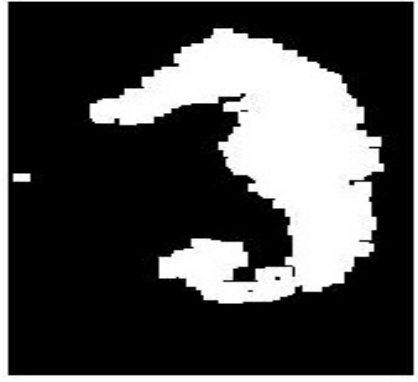
c. Output for $7 \times 7$ structuring

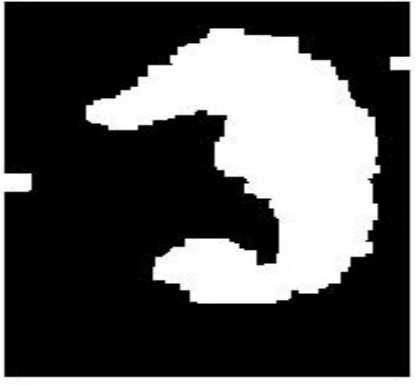

1: Outpul for $13 \times 13$ structuring element

Figure 6. a. Shows the Seahorse image. b. Shows the ground truth which is taken manually. c-f represent the output of the image with varying structuring elements.

Table 4. Correctness and Completeness values for various sizes of structuring elements for Seahorse.jpg

\begin{tabular}{|c|c|c|}
\hline $\begin{array}{c}\text { Structuring } \\
\text { Element } \\
\text { Size }\end{array}$ & $\begin{array}{c}\text { Correctnes } \\
\mathbf{s}\end{array}$ & $\begin{array}{c}\text { Completenes } \\
\mathbf{s}\end{array}$ \\
\hline $7 \times 7$ & 90.0999 & 93.7143 \\
\hline $9 \times 9$ & 85.1340 & 96.0395 \\
\hline $11 \times 11$ & 83.0496 & 96.8145 \\
\hline $13 \times 13$ & 81.0013 & 97.2830 \\
\hline $15 \times 15$ & 78.7993 & 97.5981 \\
\hline $17 \times 17$ & 76.5526 & 98.1602 \\
\hline
\end{tabular}




\section{Flamingo.jpg}

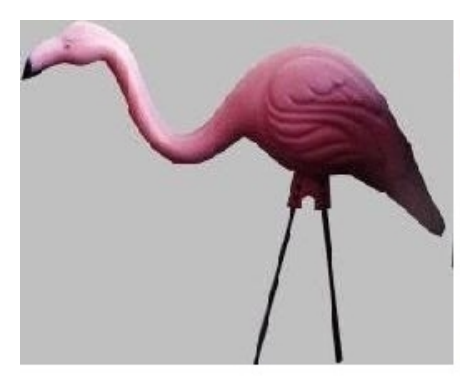

a. Original Image

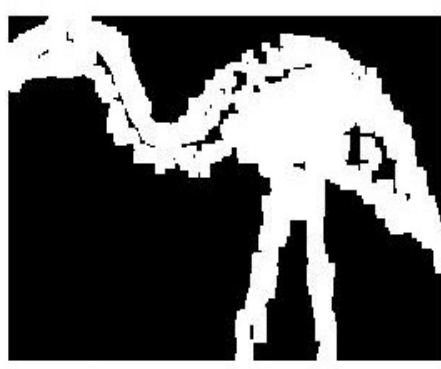

d. Ortput for $7 \mathrm{x} 7$ structuring element

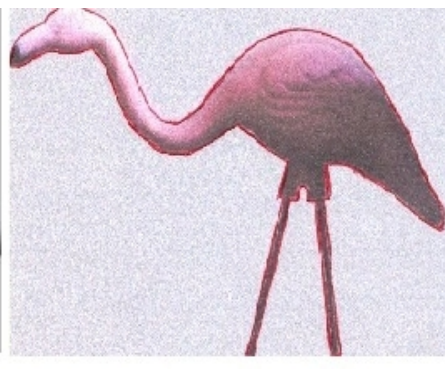

b. Ground Truth

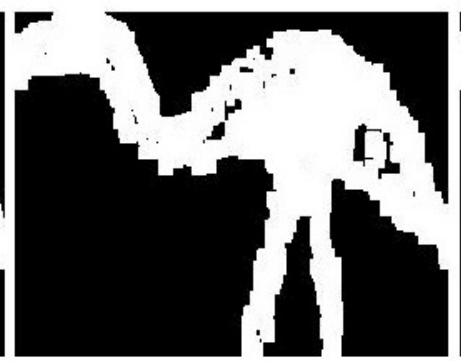

e. Output for $9 \times 9$ structuring element

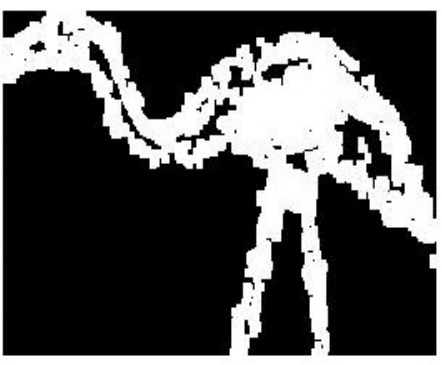

c. Ortput for $5 \times 5$ structuring element

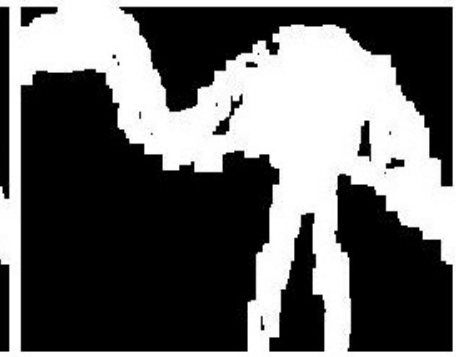

f. Output for $11 x 11$ structuring element

Figure 7. a. Shows the Flamingo image. b. Shows the ground truth which is taken manually. c-f represent the output of the image with varying structuring elements

Table 5. Correctness and Completeness values for various sizes of structuring elements for Flamingo.jpg

\begin{tabular}{|c|c|c|}
\hline $\begin{array}{c}\text { Structuring } \\
\text { Element } \\
\text { Size }\end{array}$ & $\begin{array}{c}\text { Correctnes } \\
\mathbf{s}\end{array}$ & $\begin{array}{c}\text { Completenes } \\
\mathbf{s}\end{array}$ \\
\hline $5 \times 5$ & 85.456 & 85.3091 \\
\hline $7 \times 7$ & 81.234 & 92.5348 \\
\hline $9 \times 9$ & 77.6136 & 96.1200 \\
\hline $11 \times 11$ & 74.9444 & 97.2496 \\
\hline $13 \times 13$ & 73.3578 & 97.90656 \\
\hline $15 \times 15$ & 71.6859 & 98.6985 \\
\hline
\end{tabular}


Dragonfly.jpg

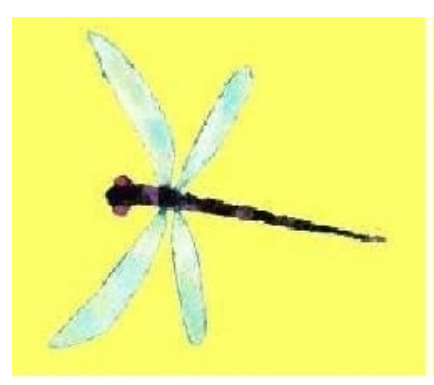

a. Original Image

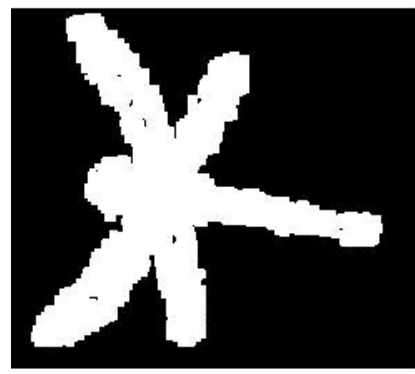

d. Ouluut lon $7 \times 57$ structuring

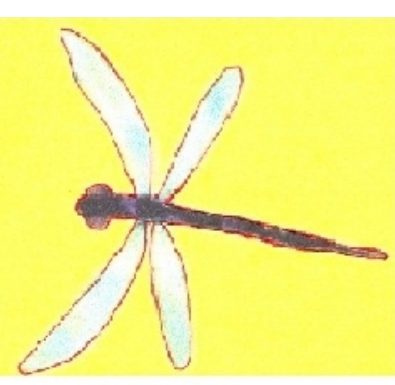

b. Groursd Truth

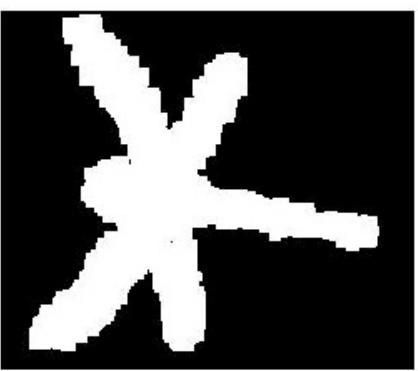

c. Output for $9 \times 9$ structuring

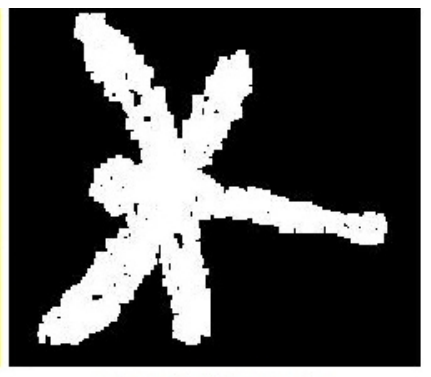

c. Output for $5 \times 5$ structuring tletueril

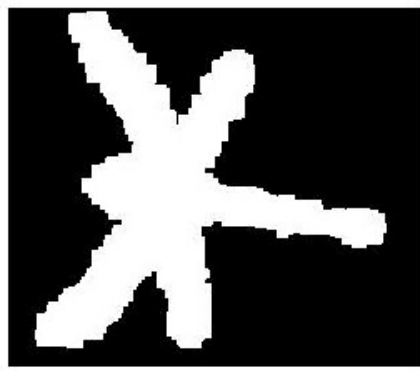

f Output for $11 \times 11$ stmothring

Figure 8. a. Shows the Dragonfly image. b. Shows the ground truth which is taken manually. c-f represent the output of the image with varying structuring elements.

Table 6. Correctness and Completeness values for various sizes of structuring elements for Dragonfly.jpg

\begin{tabular}{|c|c|c|}
\hline $\begin{array}{c}\text { Structuring } \\
\text { Element } \\
\text { Size }\end{array}$ & $\begin{array}{c}\text { Correctnes } \\
\mathbf{s}\end{array}$ & $\begin{array}{c}\text { Completenes } \\
\mathbf{s}\end{array}$ \\
\hline $5 \times 5$ & 84.6530 & 97.1433 \\
\hline $7 \times 7$ & 80.0787 & 99.3071 \\
\hline $9 \times 9$ & 76.1462 & 99.6933 \\
\hline $11 \times 11$ & 74.6419 & 99.7501 \\
\hline $13 \times 13$ & 71.6884 & 99.8012 \\
\hline $15 \times 15$ & 69.0193 & 99.8523 \\
\hline
\end{tabular}

\section{Conclusions}

A simple and regular image object segmentation method has been proposed to deal with largescale image databases. It performs dual multiscale morphological reconstruction operations on the gray levels of entire images to identify the objects. Experiments have demonstrated that OSMM yields better image object segmentation accuracy, both on shape region and boundary. The results show that the correctness and completeness of the image increases as the structuring element increases. The segmentation process can be used for identification of object in any database. 
International Journal of Computer Science, Engineering and Information Technology (IJCSEIT), Vol.3,No.4,August 2013

\section{ACKNOWLEDGEMENTS}

The authors would like to express their gratitude to Dr.K.Basavapunnaiah, President, Sri. R. Gopala Krishna, Secretary \& Correspondent, and Dr. M. Gopala Krishna, Treasurer, RVR \& JC College of Engineering for providing necessary research infrastructure. They would like to thank Dr. A. Sudhakar, Prinicipal for his invaluable suggestions and constant encouragement which led to improvise the presentation of this paper.

\section{REFERENCES}

[1] D. Comanicu and P. Meer, "Mean Shift A robust approach toward feature space ANALYSIS,” IEEE TRANS. PATTERn ANAL. MACH. INTELl., VOL. 24, NO. 5, PP. 603-619, MAY 2002.

[2] M. P. PATHEgAma AND Ö. GÖL, "EDge-END PIXEL EXTRACTION FOR EDGE BASED IMAGE SEgmentation,” In Proc. World ACAD. SCI. ENG. TeChnOl., JAN. 2005, vol. 2, PP. 164-167.

[3] Rafael C. Gonzalez and Richard E. Woods, Digital Image Processing, 2nd Edition, PEARSON EDUCATION, INC, INDIA, 2002.

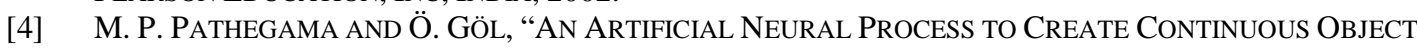
BOUNDARIES IN MEDICAL IMAGE ANALYSIS”, INTERNATIONAL SCIENTIFIC JOURNAL OF COMPUTING, VOL. 3, ISSUE 1, 2004.

[5] P. SAlEmbier and F. Marques, "Region-BASEd RePresentations of image AND VideOSEGMENTATION TOOLS FOR MULTIMEDIA SERVICES,” IEEE TRANS.CIRCUITS SYST. VIDEO TECHNOL., VOL. 9, NO. 8, PP. 1147-1169, DEC. 1999.

[6] F. Moscheni, S. BhattacharJee, and M. Kunt, "Spatio-Temporal Segmentation Based ON REGION MERGING,” IEEE TRANS. ON PATTERN ANALYSIS AND MACHINE INTELLIGENCE, VOL. 20, NO. 9, P. 897-915,1998.

[7] Y. DENG AND B. S. MANJUNATH, "UNSUPERVISED SEGMENTATION OF COLOR-TEXTURE REGIONS IN IMAGES AND VIDEO,” IEEE TRANS. PATTERn ANAL. MACh. INTELl., VOl. 23, NO. 8, PP. 800-810, AUG. 2001.

[8] L. G. Ugarriza, E. Saber, S. R. Vantaram, V. Amuso, M. Shaw, and R.Bhaskar, “Automatic IMAGE SEGMENTATION BY DYNAMIC REGION GROWTH AND MULTIRESOLUTION MERGING," IEEE TRANS. IMAGE PROCESS., VOL. 18, NO. 10, PP. 2275-2288, OCT. 2009.

[9] S. Belongie, C. Carson, H. Greenspan, and J. Malik, "Color- and teXture-based image SEGMENTATION USING EM AND ITS APPLICATION TO CONTENT-BASED IMAGE RETRIEVAL,” PROC. OF INTL. CONF. ON COMPUTER VISION, PP. 675-82, 1998.

[10] D.K. PANJWANi AND G. HEALEY, "MARKOV RANDOM FIELD MODELS FOR UNSUPERVISED SEGMENTATION OF TEXTURED COLOR IMAGES,” IEEE TRANS. ON PATTERN ANALYSIS AND MACHINE INTELLIGENCE, VOL. 17,NO.10, PP. 939-54, 1995.

[11] K. Nallaperumal, K. Krishnaveni, J. Varghese, S. Saudia, S. Annam,and P. Kumar, "A NOVEL MULTI-SCALE MORPHOLOGICAL WATERSHED SEGMENTATION ALGORITHM,” INT. J. IMAG. SCI. ENG., VOL. 1, NO.2, PP. 60-64, APR.2007.

[12] P. F. FElzENSZWAlB AND D. P. HutTENlOChER, "EFFICIENT GRAPH-BASED IMAGE SEGMENTATION," INT. J. COMPUT. VIS., VOL. 59, NO. 2, PP. 167-181, SEP. 2004.

[13] J. Shi AND J. MALiK, "Normalized CUTS AND IMAGE SEGMENTATION," IEEE TRANS. ON PATTERN ANALYSIS AND MACHINE INTELLIGENCE, VOL. 22, NO. 8, P. 888-905, 2000.

[14] S. MuKhOPADHYAY AND B. ChANDA, "Multiscale MORPHOlOGiCAl SEGMENTATION OF GRAYSCALE IMAGES,” IEEE TRANS. IMAGE PROCESS., VOL. 12, NO.5, PP. 533-549, MAY 2003.

[15] J. SERRA AND L. VINCENT, "AN OVERVIEW OF MORPHOLOGICAL FILTERING,” CiRCUITS SYST. SIGNAL PROCESS., VOL. 11, NO. 1, PP. 47-108, MAR. 1992. 